\title{
Fixed Drug Eruption Induced by Griseofulvin
}

\begin{tabular}{|l|l|l|}
\hline O. & & Boudghene-Stambouli \\
\hline A. & Mérad-Boudia \\
\hline
\end{tabular}

\section{Tlemcen}

O. Boudghene-Stambouli. A. Mérad-Boudia, Service de Dermato-Vénéréologie, Centre Hospitalo-Universitaire, 15, rue Bellevue, 13000 Tlemcen (Algeria)

Sir.

A male policeman had been treated, in February 1986, for a tinea cruris from which a Trichuphytun rubrum had been isolated. He received $1 \mathrm{~g}$ of griseofulvin daily for a month combined with topical miconazole. He came back 5 months later because the tinea cruris had reappeared. The same treatment was recommended. Forty-five days later he presented with brown pigmented macules of different sizes, round or oval on the left leg. the back and the armpits.

The examination revealed that the lesions represented a typical fixed drug eruption. The patient claimed that 'they reappeared a few hours after intake in the same places as before', i.e. during the first course of griseofulvin. We were not aware of this fact. The evolution toward pigmentation was very quick after the interruption of the treatment.

Letters to the Editor

93

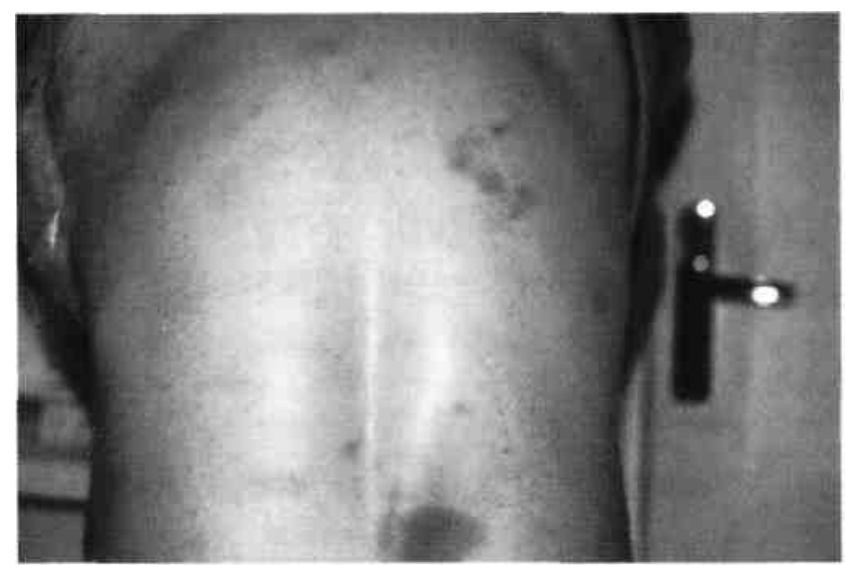




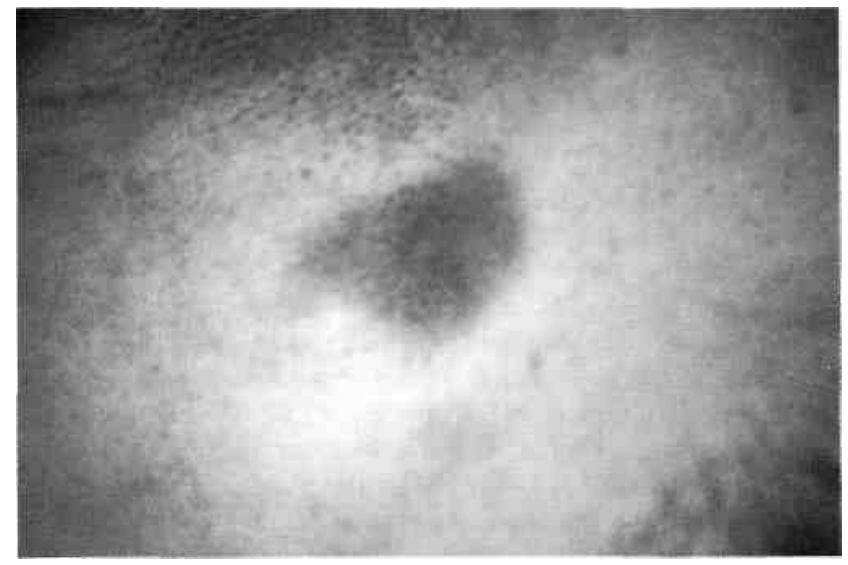

Fig. 1 Fixed drug eruption to griseofulvin. Brown pigmented macules of the back.

Fig. 2. Fixed drug eruption to griseofulvin. Brown pigmented macules of the armpit.

No general signs or involvement of the mucous membrane were noticed.

The diagnosis of fixed drug eruption due to griseofulvin was made considering the clinical aspect, the evolution, the recurrence after retaking the medication (real test of reintroduction) and the recovery after suppression of the drug. Ketoconazole at $200 \mathrm{mg}$ daily for a month healed the tinea cruris.

Comment. Griseofulvin is an antifungal widely prescribed since 1958, the first time it was used in human therapy. Side effects are usually mild. It can induce photosensitization, lupus syndromes, urticaria and gynecomasty.

Fixed drug eruption due to griseofulvin seems rare [2. 3]. The third case was reported in 1984 [1],

References

Feinstein A. Sofer E, Trau H, et al: Urticaria and fixed drug eruption in a patient treated with griseofulvin. J Am Acad Dermatol 1984;10:915-917.

Savage J: Fixed drug eruption to the griseofulvin. Br J Dermatol 1977;97:107-108.

Thyagarajan K, Kamalan A, Thombiah AS: Fixed drug eruption to the griseofulvin. Mykosen 1981;24:482-484.

Nuchal Nevus flammeus and Alopecia areata

Sir,

We read with interest the article recently published in your Journal [1] reporting on nuchal nevus flammeus (NNF) as a marker of prognosis in alopecia areata.

This study prompted us to examine for the presence of NNF 240 of our patients with alopecia areata, 126 females and 114 males, aged from 6 to 76 years $(x+S D=24.66+14.2$ years $)$ and 202 volunteers without

alopecia areata, 98 females and 104 males, aged from 3 to 76 years $(x+\mathrm{SD}=28.5+12.8)$. We grouped them for sex, age, duration of the disease, clinical type according to Ikeda's [2] classification and we classified NNF into faint $(+)$, medium $(++)$ and marked $(+++)$ since it varies in size and color [3]. Furthermore we studied the possible association between NNF and the presence of onychopathy, which is also considered a marker of the intensity of disease by some authors $[4,5]$. 
In our study NNF was confirmed more frequently in alopecic patients (53 vs. $18.2 \%$ of controls younger than 14 years and $10.8 \%$ of normal adults); more precisely NNF was present in $71.3 \%$ of the patients with alopecia totalis or universalis and in $48 \%$ of the patients with patchy alopecia, but its presence was not related to sex, age, duration and clinical type, and faint, medium and marked NNF were not related with the severity of the disease.

Moreover NNF was more frequently associated with onychopathy (60 vs. $40 \%$ ).

So the presence of NNF more than its appearance must be considered related to alopecia areata, especially that of severe degree.

The relationship between NNF and alopecia areata is an interesting matter for speculation. NNF could persist, instead of regressing with age as normally happens [6], in patients suffering or going to suffer from alopecia areata because of an altered scalp vasculature, whose involvement is also referred in this disease [7]. Moreover NNF is no more recognized as true angioma but as telangiectasia $[8,9]$ maybe, as already reported in portwine stains $[10,11]$, with neurovascular alterations and with hypotonia of the supportive dermal elements. Furthermore anagen bulb has angiogenic properties [12] and the nape area, where NNF is located, is certainly a particular site: the sensitivity to topical allergens is higher [13], the anagen period longer and, probably for this [14], the hair regrowth more difficult.

For some of these reasons a relationship between NNF and alopecia areata must be taken into account and we hope that further investigations may reveal clues as to a possible link between these two phenomena.

Giovanni Orecchia, MD

Luca Perfetti, MD

Clinica Dermatologica

Università di Pavia

P. le Golgi, 27100 Pavia (Italy) 